\title{
Beneficial effects exerted by hydroxychloroquine in treating COVID-19 patients via protecting multiple organs
}

\author{
Bo $\mathrm{Yu}^{1}$, Chenze $\mathrm{Li}^{1}$, Peng Chen ${ }^{1}, \mathrm{Jia} \mathrm{Li}^{2}$, Hualiang Jiang ${ }^{2,3^{*}}$ \& Dao-Wen Wang ${ }^{1 *}$ \\ ${ }^{1}$ Division of Cardiology, Department of Internal Medicine and Hubei Key Laboratory of Genetics and Molecular Mechanisms of Cardiological \\ Disorders, Tongji Hospital, Tongji Medical College, Huazhong University of Science and Technology, Wuhan 430030, China; \\ ${ }^{2}$ State Key Laboratory of Drug Research, Shanghai Institute of Materia Medica, Chinese Academy of Sciences, Shanghai 201203, China; \\ ${ }^{3}$ Shanghai Institute for Advanced Immunochemical Studies and School of Life Science and Technology, Shanghai Tech University, Shanghai \\ 201210, China
}

Received May 10, 2020; accepted July 25, 2020; published online August 3, 2020

Citation: Yu, B., Li, C., Chen, P., Li, J., Jiang, H., and Wang, D.W. (2021). Beneficial effects exerted by hydroxychloroquine in treating COVID-19 patients via protecting multiple organs. Sci China Life Sci 64, 330-333. https://doi.org/10.1007/s11427-020-1782-1

\section{Dear Editor,}

The coronavirus disease 2019 (COVID-19) pandemic caused by widespread infection with the severe acute respiratory syndrome coronavirus 2 (SARS-CoV-2) (Chen et al., 2020; Guan et al., 2020) has led to a global health crisis. More than 3.5 million infections and 246,838 deaths have occurred as of May 4, 2020 (Saqrane and El Mhammedi, 2020), with a rapid upward trend. Many COVID-19 patients suffer from complicated systemic injuries such as cardiac (Chen et al., 2020) and hepatic (Huang et al., 2020) damages. There are currently no specific drugs on the market to treat COVID-19-induced systemic injuries ( $\mathrm{Ni}$ et al., 2020). A study by our group has demonstrated that hydroxychloroquine (HCQ) administration dramatically reduces the fatality of critically ill COVID-19 patients (Yu et al., 2020). In the present study, we investigated whether HCQ treatment has the ability to protect multiple organs from injuries caused by SARS-CoV-2.

In this retrospective study, we extracted data of 2,882 patients (1,427 males and 1,455 females) who were clinically confirmed to have COVID-19 between February 1, 2020, and April 4, 2020, at the Tongji Hospital, Wuhan, China

\footnotetext{
*Corresponding authors (Dao-Wen Wang, email: dwwang@tjh.tjmu.edu.cn; Hualiang
} Jiang, email: hljiang@simm.ac.cn)
(IRBID: TJ-IRB20200229). The median age of all the patients was 62 (50-69) years. Of the 2,882 patients, 550 were diagnosed as critically ill and 2,332 as non-critically ill COVID-19 patients. Additionally, 278 patients (48 critical and 230 non-critical) received oral HCQ treatment (200 mg twice a day) for 7-10 days and comprised the HCQ group; the remaining 2,604 patients did not receive the HCQ treatment and comprised the NHCQ group (Figure S1 in Supporting Information). In all the HCQ-treated patients, the median time to start HCQ administration was $10(5-15)$ days after hospitalization. The baseline characteristics of the patients in these two groups are listed in Table S1 in Supporting Information. In total, of the 2,882 COVID-19 patients, 247 died (fatality rate, $8.6 \%$ ); all the deaths occurred in critically ill patients (44.9\%), including $9 / 48(18.8 \%)$ patients from the HCQ group and 238/502 (47.4\%) patients from the NHCQ group $(P<0.001)$ (Yu et al., 2020). In the HCQ group, one non-critically ill patient in whom HCQ treatment was started 7 days after hospitalization turned critically ill after receiving the treatment for more than 5 days. In the NHCQ group, 32 patients turned critically ill after spending 15 days in the hospital. The proportion of patients who turned critically ill was lower in the HCQ group than in the NHCQ group $(0.43 \%(1 / 231)$ vs. $2.48 \%(32 / 1291) ; P<0.05)$.

An inflammatory cascade and a subsequent cytokine storm form the core of COVID-19 pathophysiology that leads to 
systemic organ injury, shock, and even patient death (Henry et al., 2020; Ledford, 2020). In all the COVID-19 patients included in this study, interleukin-6 (IL-6) levels were reduced from 6.3 (2.6-25.5) $\mathrm{pg} \mathrm{mL}^{-1}$ to $3.3(1.7-7.1) \mathrm{pg} \mathrm{mL}^{-1}$ 10 days following HCQ treatment $(P<0.05)$ (Figure 1A); in non-critically ill patients, IL-6 levels were reduced significantly from $4.3(2.0-16.6) \mathrm{pg} \mathrm{mL}^{-1}$ to $3.1(1.5-8.9)$ $\mathrm{pg} \mathrm{mL} \mathrm{m}^{-1}$ in 5 days and further reached $2.9(1.6-5.2) \mathrm{pg} \mathrm{mL}^{-1}$ on the 10th day following HCQ treatment, suggesting that HCQ can reduce IL-6 levels and help maintain these low levels during the treatment period (Figure 1B, Tables S2 and S3 in Supporting Information). In contrast, there were no significant changes in IL-6 levels in the NHCQ group during the observation period. Interestingly, we noted that IL-6 levels in HCQ group were elevated to the control level after HCQ withdrawal on the 15th day following the treatment (Figure 1A and B). In critically ill patients, the IL-6 level change pattern was exactly the same as that in non-critically ill patients, with HCQ exerting a stronger lowering effect on IL-6 levels in the former (Yu et al., 2020). HCQ also reduced tumor necrosis factor-alpha and IL-2 receptor levels in both non-critically and critically ill patients during its administration period (Tables S2 and S3 in Supporting Information). There were no obvious effects on the levels of other cytokines such as IL-8 and IL-1 (Tables S2 and S3 in Supporting Information).

The liver performs vital functions such as playing a central role in metabolism, detoxification, and protein synthesis. Albumin is one of the most abundant serum proteins and is synthesized by hepatocytes; serum albumin levels are significantly reduced in COVID-19 patients (Liu C., et al., 2020; Huang et al., 2020; Liu W. et al., 2020). In our study patients, HCQ administration led to an elevation in serum albumin levels to their normal range in both the critically and non-critically ill patients (Figure $1 \mathrm{C}$ and $\mathrm{D}$ ). In the critically ill patients, HCQ treatment increased albumin levels from $32.9(27.6-36.5) \mathrm{g} \mathrm{L}^{-1}$ to $37.5(32.6-41.0) \mathrm{g} \mathrm{L}^{-1}$ on the 15 th day $(P<0.01)$. Meanwhile, in the non-critically ill patients, HCQ treatment increased albumin levels from 36.3 (32.9-40.2) $\mathrm{g} \mathrm{L}^{-1}$ to $39.3(36.5-42.5) \mathrm{g} \mathrm{L}^{-1}$ on the 15 th day $(P<0.05)$. Furthermore, HCQ treatment significantly elevated high-density lipoprotein and low-density lipoprotein levels (Tables S2 and S3 in Supporting Information). These data suggest that SARS-CoV-2 infection leads to liver dysfunction that affects protein synthesis, even though the levels of usual liver function markers such as aspartate aminotransferase and alanine aminotransferase are not elevated (Tables S2 and S3 in Supporting Information). HCQ treatment may also reduce hepatotoxicity, and it improves hepatocyte protein synthesis function.

Cardiac troponin I (cTnI) and amino-terminal pro-brain natriuretic peptide (NT-proBNP) are cardiac biomarkers, and their elevated levels indicate cardiac injury and cardiac dysfunction, which are common in COVID-19 patients, especially in critically ill patients (Guo et al., 2020). In the critically ill patients in the HCQ group, HCQ reduced NTproBNP levels from $1,070.0(418.0-6,691.0) \mathrm{pg} \mathrm{mL}^{-1}$ to $517.0(88.0-936.0) \mathrm{pg} \mathrm{mL}^{-1}(P=0.082) 10$ days after oral use, and the levels further reached $203.0(41.0-865.0) \mathrm{pg} \mathrm{mL}^{-1}$ $(P<0.05)$ on the 20th day. In the critically ill patients in the NHCQ group, NT-proBNP levels even elevated gradually (Figure 1E; Table S2 in Supporting Information). In the critically ill patients in the HCQ group, HCQ treatment decreased cTnI levels from $14.5(2.9-30.9) \mathrm{pg} \mathrm{mL}^{-1}$ to 7.1 (4.0-30.8) $\mathrm{pg} \mathrm{mL}^{-1} 10$ days after oral use, and the levels reached $5.3(2.3-17.4) \mathrm{pg} \mathrm{mL}^{-1}$ on the 20th day (Figure $1 \mathrm{G}$; Table S2 in Supporting Information). HCQ treatment had no effect on cTnI and NT-proBNP levels in the non-critically ill COVID-19 patients because in almost all of these patients, cTnI and NT-proBNP levels were below the upper limit of the normal range (Figure 1F and H; Table S3 in Supporting Information).

We also found that in all the HCQ group patients, fatality rate in patients with cardiac injury ( $\mathrm{cTnI}>34.2 \mathrm{pg} \mathrm{mL}^{-1}$ on admission) was $29.4 \%(5 / 17)$, whereas in all the NHCQ group patients, fatality rate in patients with cardiac injury was $52.5 \%$ (94/179). In the HCQ group patients with cardiac injury on admission, HCQ treatment decreased cTnI levels from 95.7 (69.3-273.8) $\mathrm{pg} \mathrm{mL}^{-1}$ to $24.3(14.2-65.8) \mathrm{pg} \mathrm{mL}^{-1}$ 5 days after oral use, and in the NHCQ group patients with cardiac injury on admission, $\mathrm{cTnI}$ levels were reduced from $109.6(53.8-478.7) \mathrm{pg} \mathrm{mL}^{-1}$ to $39.7(10.8-169.6) \mathrm{pg} \mathrm{mL}^{-1}$ during the same period. These analyses support our hypothesis that HCQ treatment can reduce the fatality of COVID-19 patients via alleviating cardiac injuries.

Furthermore, we analyzed the effect of HCQ treatment initiation time on therapeutic response and found that HCQ treatment started early (within 5 days of admission) resulted in a lower fatality rate than that started after 5 days of admission $(1 / 73(1.4 \%)$ vs. $8 / 205(3.9 \%))$, although the difference is not significant $(P>0.05)$. However, HCQ treatment started early (within 5 days of admission) exerted a better effect in reducing IL-6 levels than that started 5 days after admission (2.6 (1.5-4.5) $\mathrm{pg} \mathrm{mL}^{-1}$ vs. $3.7(1.9-8.5) \mathrm{pg} \mathrm{mL}^{-1}$ on the 10th day of treatment; $P<0.05)$. Similarly, HCQ treatment started early exerted a better effect in elevating albumin levels than that started 5 days after admission (40.5 $(35.0-42.6) \mathrm{g} \mathrm{L}^{-1}$ in the HCQ group vs. 35.7 (32.5-39.5) $\mathrm{g} \mathrm{L}^{-1}$ in the NHCQ group on the 5 th day following treatment; $P<0.05)$ and $\left(39.8(35.4-42.4) \mathrm{g} \mathrm{L}^{-1}\right.$ in the HCQ group vs. $37.4(33.3-39.6) \mathrm{g} \mathrm{L}^{-1}$ in the NHCQ group on the 10th day following treatment; $P<0.05$ ). These results suggest that HCQ treatment should be started early following hospitalization.

In summary, this retrospective study with its large sample size demonstrated that a cytokine storm in patients with 

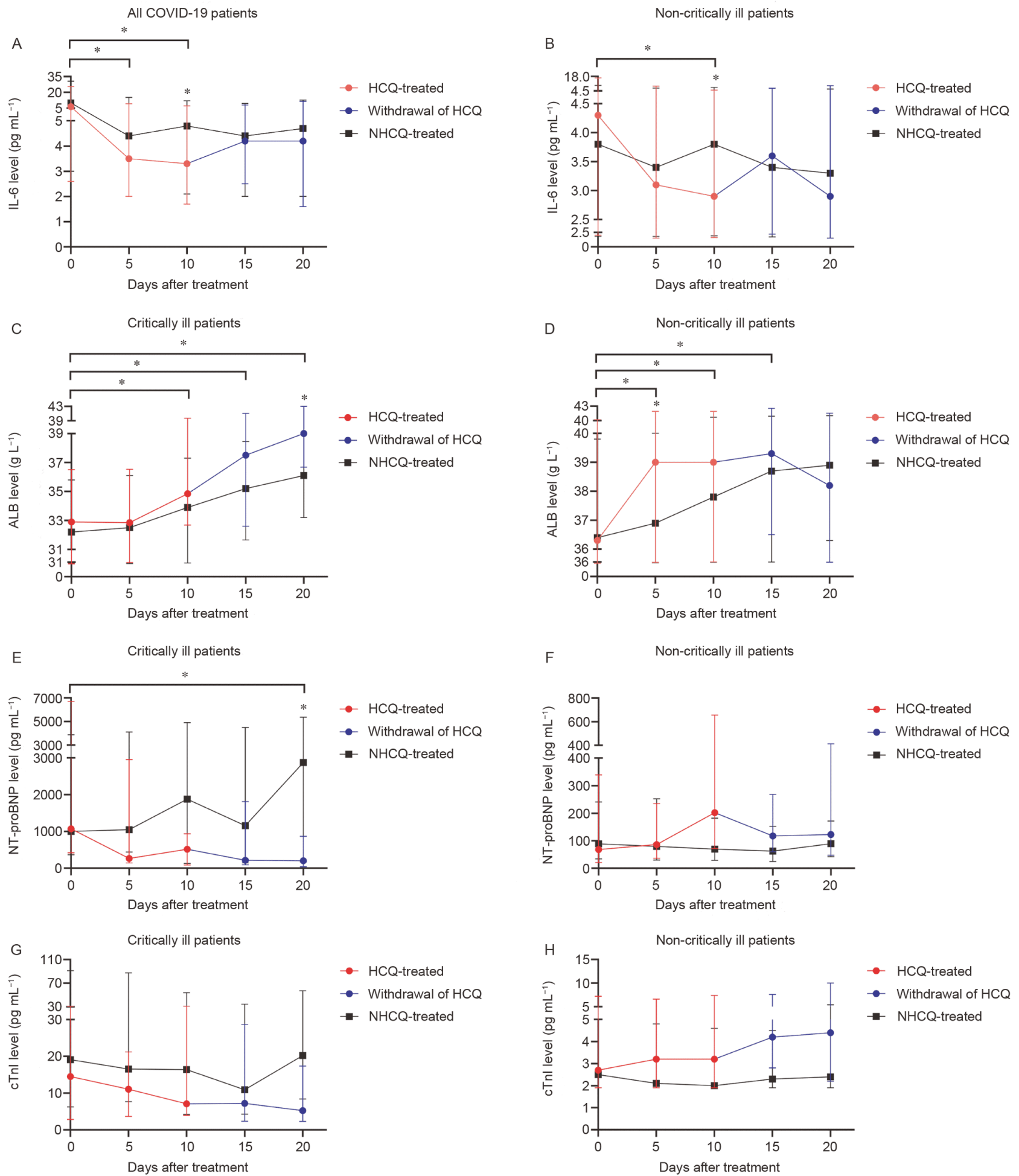

Figure 1 HCQ treatment exerted some changes. A, In all the COVID-19 patients, IL-6 levels significantly reduced after HCQ treatment, $* P<0.05$. IL- 6 levels were elevated after HCQ withdrawal in the HCQ group patients. B, In the non-critically ill patients, IL-6 levels were significantly reduced after 10 days of HCQ treatment, $* P<0.05$. C, In critically ill patients, albumin levels were significantly elevated after HCQ treatment, $* P<0.05$. D, In the non-critically ill patients, albumin levels were also significantly elevated after HCQ treatment, $* P<0.05$. E, In the critically ill patients treated with HCQ, NT-proBNP levels were significantly reduced, ${ }^{*} P<0.05$, whereas in the NHCQ group patients, NT-proBNP levels were elevated. F, In almost all the non-critically ill patients, NT-proBNP levels were below the upper limit of the normal range. G, In the critically ill patients treated with HCQ, cTnI levels were reduced; furthermore, cTnI levels of the HCQ group patients were significantly lower than those of the NHCQ group patients after 20 days of HCQ treatment, $* P<0.05$. H, In almost all the non-critically ill patients, cTnI levels were below the upper limit of the normal range. Data are presented as medians and interquartile ranges (Q1-Q3). HCQ, hydroxychloroquine; NHCQ, non-hydroxychloroquine; IL, interleukin; ALB, albumin; NT-proBNP, amino-terminal pro-brain natriuretic peptide; cTnI, cardiac troponin I. 
COVID-19 causes injuries to multiple organs, including the heart and liver among others. However, HCQ treatment can reduce systemic inflammation and inhibit the cytokine storm, thus protecting multiple organs from inflammatory injuries, such as detoxification in the liver and attenuation of cardiac injury. Although there are some limitations of this study such as small number of patients in the HCQ group and several different HCQ treatment initiation times, we can still propose an HCQ regimen for treating COVID-19: the treatment should be started as early as possible at low dosage, i.e., $200 \mathrm{mg}$ twice a day for 7-10 days or more until the illness is markedly alleviated, and then the dose should be reduced to $200 \mathrm{mg}$ per day until the patient is cured or discharged.

Compliance and ethics The author(s) declare that they have no conflict of interest.

Acknowledgements We thank all medical staff and medical data managers for data extraction and help for analysis. We would like to thank Prof. Eric H. Xu at Shanghai Institute of Materia Medica, Chinese Academy of Sciences, for his useful suggestion and language improvement in writing this paper. This work was supported in part by the National Key R\&D Program of Ministry of Science and Technology of China (2020YFC0844500), National Natural Science Foundation of China (31130031), Emergency Project Fund of Chinese Academy of Sciences (2020YJFK0105), and Chinese Academy of Engineering and Ma Yun Foundation (2020-CMKYGG-05).

\section{References}

Chen, C., Chen, C., Yan, J.T., Zhou, N., Zhao, J.P., and Wang, D.W. (2020). Analysis of myocardial injury in patients with COVID-19 and association between concomitant cardiovascular diseases and severity of COVID-19 (in Chinese). Zhonghua Xin Xue Guan Bing Za Zhi 48, E008.
Guan, W.J., Ni, Z.Y., Hu, Y., Liang, W.H., Ou, C.Q., He, J.X., Liu, L., Shan, H., Lei, C.L., Hui, D.S.C., et al. (2020). Clinical characteristics of coronavirus disease 2019 in China. N Engl J Med 382, 1708-1720.

Guo, T., Fan, Y., Chen, M., Wu, X., Zhang, L., He, T., Wang, H., Wan, J., Wang, X., and Lu, Z. (2020). Cardiovascular implications of fatal outcomes of patients with coronavirus disease 2019 (COVID-19). JAMA Cardiol 5, 811 .

Henry, B.M., de Oliveira, M.H.S., Benoit, S., Plebani, M., and Lippi, G. (2020). Hematologic, biochemical and immune biomarker abnormalities associated with severe illness and mortality in coronavirus disease 2019 (COVID-19): a meta-analysis. Clin Chem Lab Med 58, 1021-1028.

Huang, W., Li, C., Wang, Z., Wang, H., Zhou, N., Jiang, J., Ni, L., Zhang, X.A., and Wang, D.W. (2020). Decreased serum albumin level indicates poor prognosis of COVID-19 patients: hepatic injury analysis from 2,623 hospitalized cases. Sci China Life Sci https://doi.org/10.1007/ s11427-020-1733-4.

Ledford, H. (2020). How does COVID-19 kill? Uncertainty is hampering doctors' ability to choose treatments. Nature 580, 311-312.

Liu, C., Jiang, Z.C., Shao, C.X., Zhang, H.G., Yue, H.M., Chen, Z.H., Ma, B.Y., Liu, W.Y., Huang, H.H., Yang, J., et al. (2020). Preliminary study of the relationship between novel coronavirus pneumonia and liver function damage: a multicenter study (in Chinese). Zhonghua Gan Zang Bing Za Zhi 28, 148-152.

Liu, W., Tao, Z.W., Wang, L., Yuan, M.L., Liu, K., Zhou, L., Wei, S., Deng, Y., Liu, J., Liu, H.G., et al. (2020). Analysis of factors associated with disease outcomes in hospitalized patients with 2019 novel coronavirus disease. Chin Med J 133, 1032-1038.

Ni, L., Zhou, L., Zhou, M., Zhao, J., and Wang, D.W. (2020). Combination of western medicine and Chinese traditional patent medicine in treating a family case of COVID-19. Front Med 14, 210-214.

Saqrane, S., and El Mhammedi, M.A. (2020). Review on the global epidemiological situation and the efficacy of chloroquine and hydroxychloroquine for the treatment of COVID-19. New Microb New Infect 35, 100680.

Yu, B., Li, C., Chen, P., Zhou, N., Wang, L., Li, J., Jiang, H., and Wang, D. W. (2020). Low dose of hydroxychloroquine reduces fatality of critically ill patients with COVID-19. Sci China Life Sci https://doi. org/10.1007/s11427-020-1732-2.

\section{SUPPORTING INFORMATION}

The supporting information is available online at https://doi.org/10.1007/s11427-020-1782-1. The supporting materials are published as submitted, without typesetting or editing. The responsibility for scientific accuracy and content remains entirely with the authors. 